\title{
Anomalies and symmetries of the regularized action
}

\author{
César D. Fosco a,1, Francisco D. Mazzitelli b,2 \\ ${ }^{a}$ Centro Atómico Bariloche, 8400 Bariloche, Argentina \\ ${ }^{\mathrm{b}}$ Departamento de Fí sica, Facultad de Ciencias Exactas y Naturales, Universidad de Buenos Aires - Ciudad Universitaria, \\ Pabellón I, 1428 Buenos Aires, Argentina
}

Received 21 March 2000; received in revised form 3 April 2000; accepted 3 April 2000

Editor: H. Georgi

\begin{abstract}
We show that the Pauli-Villars regularized action for a scalar field in a gravitational background in $1+1$ dimensions has, for any value of the cutoff $M$, a symmetry which involves non-local transformations of the regulator field plus (local) Weyl transformations of the metric tensor. These transformations, an extension to the regularized action of the usual Weyl symmetry transformations of the classical action, lead to a new interpretation of the conformal anomaly in terms of the (non-anomalous) Jacobian for this symmetry. Moreover, the Jacobian is automatically regularized, and yields the correct result when the masses of the regulators tend to infinity. In this limit the transformations, which are non-local on a scale of $\frac{1}{M}$, become the usual Weyl transformations of the metric. We also present the example of the chiral anomaly in $1+1$ dimensions, showing that the Pauli-Villars regularized action has a non-local symmetry. This symmetry is similar to the one of (lattice) Ginsparg-Wilson fermions, with the ultraviolet cutoff playing the role of the inverse of the lattice spacing. (C) 2000 Published by Elsevier Science B.V. All rights reserved.
\end{abstract}

\section{Introduction}

Anomalies are one of the most striking manifestations of the presence of ultraviolet infinities in quantum field theory phenomena. They have important consequences for the quantum consistency (unitarity and renormalizability) of different models, as well as for the study of the theoretical structure of quantum field theory, providing interesting relations with mathematical objects.

The regularization of a theory is a procedure with, by its very definition, produces a violent modification in its large momentum behaviour. Anomalies

\footnotetext{
${ }^{1}$ E-mail: fosco@cab.cnea.gov.ar

${ }^{2}$ E-mail: fmazzi@df.uba.ar
}

arise when this modification, whatever the particular regularization method applied, violates a classical symmetry. In the resulting renormalized quantum field theory, the quantum counterpart of that symmetry becomes 'anomalous'. In particular, continuum symmetries will no longer lead to the conservation of a current, thus modifying the naive Ward identities.

In the functional integral representation, anomalies are usually attributed to the fact that, while the classical action is invariant under a given symmetry transformation, the integration measure is not. When performing a change of variables associated to the classical symmetry, the Jacobian of the transformation is, due to ultraviolet divergences, not well defined. When carefully evaluated, by introducing a proper regularization, this Jacobian gives rise to an 
anomalous term in the effective action [1], or, depending on the context, to anomalous Ward identities.

In this approach, the symmetry transformations are implemented on the unregularized action, which is formally invariant, and the regularization is only implemented afterwards, when computing the Jacobian ${ }^{3}$ of the transformation. It is worth remarking that the unregularized action contains field components at all the momentum scales, in particular the ones that are above the cutoff. To avoid this unpleasant feature, we shall start by considering the regularized action, and restrict our study to transformations that leave this functional invariant. Of course, we shall impose the constraint that the resulting transformations should tend to the usual ones when the cutoff is removed. Also, due to the fact that they avoid the large momentum modes, they will necessarily have to be non local, although in a scale of the order of the inverse of the cutoff.

As a particularly convenient form of the regularized action, which we will use in this note, we shall introduce a set of Pauli-Villars regulator fields, with carefully tuned masses $M_{i}$ that tend to infinity at the end of the calculation. Due to the presence of the regulator masses, the regularized action will be no longer invariant under some the classical symmetries, namely, those that rely on the masslessness of the fields in the action.

In the present letter, we will point out that this regularized action does have a generalized, non local symmetry, that reproduces the classical symmetry in the limit $M_{i} \rightarrow \infty$. Moreover, when performing a change of variables in the path integral based on this symmetry, the associated Jacobian is finite, reproducing the anomalous term in the effective action, when the cutoff tends to infinity.

In Section 2 we discuss this procedure in detail for the gravitational conformal anomaly of a massless real scalar field in $1+1$ dimensions. We exhibit the explicit form of the non local symmetry of the regularized action and compute the conformal anomaly, which of course agrees with the Liouville action [2,3]. In Section 3 we consider massless

\footnotetext{
${ }^{3}$ The regularization of this Jacobian is of course related to the definition of the integration measure.
}

fermions in $1+1$ dimensions coupled to the electromagnetic field. Again we present a generalized chiral symmetry of the regularized action and compute the chiral anomaly. Section 4 contains our conclusions.

\section{The conformal anomaly in $1+1$ dimensions}

In this section we shall consider the partition function $\mathscr{Z}\left[g_{\mu \nu}\right]$, corresponding to a massless scalar field in the presence of an external gravitational background in $1+1$ dimensions:

$\mathscr{Z}\left[g_{\mu \nu}\right]=\int[\mathscr{D} \varphi]_{g} e^{i S\left[\varphi, g_{\mu \nu}\right]}$

where

$S\left[\varphi, g_{\mu \nu}\right]=\frac{1}{2} \int d^{2} x \sqrt{-g} g^{\mu \nu} \partial_{\mu} \varphi \partial_{\nu} \varphi$

and $g=\operatorname{det}\left(g_{\mu \nu}\right)$. In Eq. (1) we have made it explicit the fact that the definition of the scalar field integration measure depends upon the background metric $g_{\mu \nu}$.

The classical action $S\left[\varphi, g_{\mu \nu}\right]$ is invariant under Weyl transformations of the metric:

$g_{\mu \nu} \rightarrow g_{\mu \nu}^{\omega}(x)=\omega(x) g_{\mu \nu}(x)$

$S\left[\varphi, g_{\mu \nu}^{\omega}\right]=S\left[\varphi, g_{\mu \nu}\right]$

for any (strictly positive) $\omega(x)$. In adequate coordinates, any metric in two dimensions is conformally flat:

$g_{\mu \nu}=e^{\sigma(x)} \eta_{\mu \nu}$

where $\eta_{\mu \nu}=\operatorname{diag}(1,-1)$ denotes the flat Minkowski metric. The classical dynamics of a scalar field in a gravitational background in $1+1$ dimensions is therefore trivial:

$S\left[\varphi, g_{\mu \nu}\right]=S\left[\varphi, \eta_{\mu \nu}\right]=\frac{1}{2} \int d^{2} x \eta^{\mu \nu} \partial_{\mu} \varphi \partial_{\nu} \varphi$.

This conclusion does not hold true, of course, for the quantum dynamics, the reason being the existence of the conformal or trace anomaly, which spoils the symmetry under the transformations (3) and produces a non vanishing trace in the energy momentum tensor [4].

In the usual setting, one derives the quantum effects by dealing with the vacuum functional (1). 
Under a Weyl transformation (3), and using the property (4), we see that

$\mathscr{Z}\left[g_{\mu \nu}^{\omega}\right]=\int[\mathscr{D} \varphi]_{g^{\omega}} e^{i S\left[\varphi, g_{\mu \nu}\right]}$.

Namely, any possible quantum effect must come from the non-invariance of the integration measure under Weyl transformations of the metric.

As shown by Polyakov [2,3], there is, indeed, an anomalous Jacobian, the exponential of the Liouville action

$$
\begin{aligned}
{[\mathscr{D} \varphi]_{g}{ }^{\omega} } & =[\mathscr{D} \varphi]_{g} J[\sigma] \\
& =[\mathscr{D} \varphi]_{g} \exp i \mathscr{A}\left[g_{\mu \nu}, w\right]
\end{aligned}
$$

where $\mathscr{A}\left[g_{\mu \nu}, w\right]$ is the anomaly, a local functional of $g_{\mu \nu}$.

By using the decomposition (5), and normalizing $\mathscr{Z}$ such that $\mathscr{Z}\left[\eta_{\mu \nu}\right]=1$, one also sees that

$\mathscr{Z}\left[g_{\mu \nu}\right]=J[\sigma]=\exp i \mathscr{A}[w]$,

where $\mathscr{A}[w] \equiv \mathscr{A}\left[\eta_{\mu \nu}, w\right]$ is given by

$\mathscr{A}[w]=\frac{1}{96 \pi} \int d^{2} x \frac{1}{w^{2}} \partial_{\mu} w \partial^{\mu} w$.

As can be easily proved from the perturbative derivation of the anomaly given in Ref. [3], when the system consists of $d$ bosonic scalar fields and $\bar{d}$ Grassmann scalar fields the Jacobian becomes $J[\sigma]$ $=\exp i(d-\bar{d}) \mathscr{A}[g, w]$.

In what follows we will show that, if one works with the regularized action rather than the classical action $S[\varphi, g]$, there is a non local symmetry, and one can compute the anomaly as the Jacobian associated to a non local redefinition of the fields. The Pauli-Villars method, first applied to this system in Ref. [5], amounts to the introduction of three 'regulator fields' $\bar{\chi}, \chi, \eta$ and a cutoff $M$ so that

$$
\begin{aligned}
S^{\mathrm{reg}} & {\left[\varphi, \bar{\chi}, \chi, \eta, g_{\mu \nu}, M\right] } \\
& =\frac{1}{2} \int d^{2} x \sqrt{-g}\left(g^{\mu \nu} \partial_{\mu} \varphi \partial_{\nu} \varphi+g^{\mu \nu} \partial_{\mu} \bar{\chi} \partial_{\nu} \chi\right. \\
& \left.-M^{2} \bar{\chi} \chi+g^{\mu \nu} \partial_{\mu} \eta \partial_{\nu} \eta-2 M^{2} \eta^{2}\right)
\end{aligned}
$$

where $\bar{\chi}, \chi$ are complex Grassmann fields, while $\eta$ is a real scalar field. The number and masses of the regulators are just right to make the vacuum energy in a non-trivial background finite. For the sake of convenience, we rewrite (11) in a more compact form as follows:

$$
\begin{aligned}
& S^{\mathrm{reg}}\left[\varphi, \bar{\chi}, \chi, \eta, g_{\mu \nu}, M\right] \\
& =-\frac{1}{2}\left[\left\langle\varphi\left|\square^{\prime}\right| \varphi\right\rangle+\left\langle\bar{\chi}\left|\left(\square^{\prime}+M^{2} \sqrt{-g}\right)\right| \chi\right\rangle\right. \\
& \left.\quad+\left\langle\eta\left|\left(\square^{\prime}+2 M^{2} \sqrt{-g}\right)\right| \eta\right\rangle\right]
\end{aligned}
$$

where

$$
\square^{\prime}=\sqrt{-g} \square=\partial_{\mu} \sqrt{-g} g^{\mu \nu} \partial_{\nu}
$$

and $\square$ is the usual curved space Laplacian operator $\square=\frac{1}{\sqrt{-g}} \partial_{\mu} \sqrt{-g} g^{\mu \nu} \partial_{\nu}$. We used a Dirac bracket like notation for the scalar product in flat two dimensional spacetime:

$$
\left\langle\phi_{1} \mid \phi_{2}\right\rangle=\int d^{2} x \phi_{1}(x) \phi_{2}(x),
$$

which is convenient, since we have absorbed the factor $\sqrt{-g}$ of the measure in the operators. It is worth noting that both $\square^{\prime}$ and $\sqrt{-g}$ are symmetric (real Hermitian) operators for the scalar product (14). Also, $\square^{\prime}$ is explicitly invariant under Weyl transformations.

Due to the presence of the mass terms for the regulator fields, it is evident that the regularized action is not invariant under Weyl transformations of the metric tensor:

$$
\begin{aligned}
S^{\mathrm{reg}}[ & \left.\varphi, \bar{\chi}, \chi, \eta, g_{\mu \nu}^{\omega}, M\right] \\
= & -\frac{1}{2}\left[\left\langle\varphi\left|\square^{\prime}\right| \varphi\right\rangle\right. \\
& +\left\langle\bar{\chi}\left|\left(\square^{\prime}+M^{2} \omega \sqrt{-g}\right)\right| \chi\right\rangle \\
& \left.+\left\langle\eta\left|\left(\square^{\prime}+2 M^{2} \omega \sqrt{-g}\right)\right| \eta\right\rangle\right] \\
\neq & S^{\mathrm{reg}}\left[\varphi, \bar{\chi}, \chi, \eta, g_{\mu \nu}, M\right] .
\end{aligned}
$$

We may, however, compensate the non invariance of $S^{\text {reg }}$ by a transformation of the regulator fields:

$$
\begin{aligned}
\left|\varphi^{w}\right\rangle= & |\varphi\rangle, \\
\left|\eta^{w}\right\rangle= & \left(\square^{\prime}+2 M^{2} w \sqrt{-g}\right)^{-1 / 2} \\
& \times\left(\square^{\prime}+2 M^{2} \sqrt{-g}\right)^{1 / 2} \mid \eta>, \\
\mid \chi^{w}>= & \left(\square^{\prime}+M^{2} w \sqrt{-g}\right)^{-1 / 2} \\
& \times\left(\square^{\prime}+M^{2} \sqrt{-g}\right)^{1 / 2} \mid \chi>
\end{aligned}
$$


after which we see that

$$
S_{\text {reg }}\left[\varphi^{w}, \eta^{w}, \bar{\chi}^{w}, \chi^{w}, g^{w}, M\right]=S_{\text {reg }}[\varphi, \eta, \bar{\chi}, \chi, g, M] .
$$

Eqs. (16) and (17) define the non local symmetry of the regularized action. This symmetry must be studied of course at the quantum level, by considering the regularized vacuum functional

$$
\begin{aligned}
Z_{\mathrm{reg}}\left[g_{\mu \nu}\right]= & \int[\mathscr{D} \varphi \mathscr{D} \eta \mathscr{D} \bar{\chi} \mathscr{D} \chi]_{g} \\
& \times \exp \left(i S_{\mathrm{reg}}[\varphi, \eta, \bar{\chi}, \chi, g, M]\right) .
\end{aligned}
$$

Performing the Weyl transformation (3) for $g_{\mu \nu}$ in (18) followed by the change of variables (16) in the functional integral, we see that:

$$
\begin{aligned}
Z_{\text {reg }}\left[g_{\mu \nu}^{w}\right]= & \int\left[\mathscr{D} \varphi^{w} \mathscr{D} \eta^{w} \mathscr{D} \bar{\chi}^{w} \mathscr{D} \chi^{w}\right]_{g^{w}} \\
& \times \exp \left(i S_{\text {reg }}[\varphi, \eta, \bar{\chi}, \chi, g, M]\right) \\
= & J_{\text {reg }}[g, w, M] \int[\mathscr{D} \varphi \mathscr{D} \eta \mathscr{D} \bar{\chi} \mathscr{D} \chi]_{g} \\
& \times \exp \left(i S_{\text {reg }}[\varphi, \eta, \bar{\chi}, \chi, g, M]\right)
\end{aligned}
$$

where $J_{\text {reg }}$ denotes the Jacobian for the transformation (16):

$$
\begin{aligned}
J_{\text {reg }}[g, w, M]= & \operatorname{det}\left[\left(\square^{\prime}+M^{2} \sqrt{-g}\right)^{-1}\right. \\
& \left.\times\left(\square^{\prime}+M^{2} w \sqrt{-g}\right)\right] \\
& \times \operatorname{det}\left[\left(\square^{\prime}+2 M^{2} \sqrt{-g}\right)^{-1}\right. \\
& \left.\times\left(\square^{\prime}+2 M^{2} w \sqrt{-g}\right)\right]^{-1 / 2},
\end{aligned}
$$

and the suffix 'reg' is used because, as we will see now, this Jacobian is finite. It is worth noting that, although the integration measure for each field [ $\left.\mathscr{D} \phi_{i}\right]$ depends non trivially on the background metric, as in the unregularized case, the product of the integration measures for the four fields does not depend on $w$, due to the cancellation between the anomalous factors corresponding to bosonic and Grassmann fields.
These Jacobian factors are independent of the masses of the fields.

If we define the finite quantities $Z$ and $J$ as the limit of $Z_{\text {reg }}$ and $J_{\text {reg }}$ for $M \rightarrow \infty$ Eq. (19) implies that $Z\left[g^{w}\right]=J[g, w] Z[g]$. Thus we have to evaluate Eq. (20) in the limit $M \rightarrow \infty$.

Let us now calculate the regulated Jacobian (20). As the metric is assumed to be conformally flat, and $\square^{\prime}$ is invariant under (3), it is obvious that we may replace $\square^{\prime}$ by $\square$, and that $\sqrt{-g}=1$. We then rewrite $J_{\text {reg }}$ in the form:

$J_{\text {reg }}[\eta, w, M]=J_{\text {reg }}^{(1)}[w, M] \times J_{\text {reg }}^{(2)}[w, M]$

where

$J_{\text {reg }}^{(1)}[w, M]=\operatorname{det}\left[\left(\square+M^{2}\right)^{-1}\left(\square+M^{2} w\right)\right]$,

$J_{\text {reg }}^{(2)}[w, M]=\left[J_{\text {reg }}^{(1)}[w, \sqrt{2} M]\right]^{-1 / 2}$.

We shall now, for calculational purposes, consider $J_{\text {reg }}^{(1)}$ alone, since the factor $J_{\text {reg }}^{(2)}$ can be obtained from it by some simple substitutions. However, the two factors have to be taken together for the cancellation between UV divergences to happen.

To evaluate $J_{\text {reg }}^{(1)}[w, M]$, we take into account the fact that we will, in the end, be interested in the $M \rightarrow \infty$ limit. This justifies the use of some form of expansion in powers of $\frac{1}{M}$. A small dimensionless parameter has then to be built using $M$, and the only other dimensionfull object: derivatives of $\omega(\omega$ itself is dimensionless). We then follow the derivative expansion technique [6] to split the field $\omega$ into a slowly varying part $\tilde{\omega}$ plus a fluctuating piece $\alpha$

$\omega(x)=\tilde{\omega}(x)+\alpha(x)$,

where $\tilde{\omega}(x)$ is to be regarded as a constant when acted by the derivative operator. We then rotate to Euclidean spacetime, and expand $\ln J_{\text {reg }}^{(1)}[w, M]$ in powers of $\alpha$, starting from

$$
\begin{aligned}
\ln J_{\text {reg }}^{(1)}[w, M]= & \operatorname{Tr} \ln \left(\frac{-\partial^{2}+M^{2} \tilde{\omega}}{-\partial^{2}+M^{2}}\right) \\
& +\operatorname{Tr} \ln \left(1+\frac{M^{2} \alpha}{-\partial^{2}+M^{2} \tilde{\omega}}\right),
\end{aligned}
$$

where $\partial^{2}=\delta_{\mu \nu} \partial_{\mu} \partial_{\nu}$ is the flat, Euclidean spacetime Laplace operator. Taking into account that the linear 
term vanishes, and terms with more than two $\alpha$ 's are suppressed by negative powers of $M$, it is sufficient to use the expansion:

$$
\begin{aligned}
\ln J_{\text {reg }}^{(1)}[w, M]= & \operatorname{Tr} \ln \left(\frac{-\partial^{2}+M^{2} \tilde{\omega}}{-\partial^{2}+M^{2}}\right) \\
& -\frac{1}{2} \operatorname{Tr}\left(\frac{M^{2} \alpha}{-\partial^{2}+M^{2} \tilde{\omega}} \frac{M^{2} \alpha}{-\partial^{2}+M^{2} \tilde{\omega}}\right) \\
& +\mathscr{O}\left(\frac{1}{M^{2}}\right) .
\end{aligned}
$$

The first, zero derivative term, is divergent (even for finite $M$ ). However, remembering that we are using a Pauli-Villars scheme, we have to evaluate the momentum integral that results from combining it with the corresponding contribution from $\ln J^{(2)}$. This produces a finite answer:

$$
\begin{aligned}
& \operatorname{Tr} \ln \left(\frac{-\partial^{2}+M^{2} \tilde{\omega}}{-\partial^{2}+M^{2}}\right)-\frac{1}{2} \operatorname{Tr} \ln \left(\frac{-\partial^{2}+2 M^{2} \tilde{\omega}}{-\partial^{2}+2 M^{2}}\right) \\
& \quad=\frac{M^{2} \ln 2}{4 \pi}(\tilde{\omega}-1),
\end{aligned}
$$

proportional to $M^{2}$. This is finite (for finite $M$ ), and this shows that the Jacobian for the non local symmetry transformations is indeed finite. Of course, when $M$ tends to infinite, this contribution diverges. The term proportional to $\tilde{\omega}$ requires the introduction of a counterterm of the cosmological constant type. The $\tilde{\omega}$-independent divergence in Eq. (26) can be absorbed into the normalization factor of $\mathscr{Z}$.

For the second order term (which is finite when $M \rightarrow \infty$ ), a standard calculation yields, for $M \rightarrow \infty$

$$
\begin{aligned}
& -\frac{1}{2} \operatorname{Tr}\left[\frac{M^{2}}{-\partial^{2}+M^{2} \tilde{\omega}} \alpha \frac{M^{2}}{-\partial^{2}+M^{2} \tilde{\omega}} \alpha\right] \\
& =-\frac{1}{48 \pi} \int d^{2} x \frac{1}{\tilde{w}^{2}} \partial_{\mu} \alpha \partial^{\mu} \alpha .
\end{aligned}
$$

The derivative expansion technique implies [6], on the other hand, that $\tilde{\omega}$ may be replaced by $\omega$ in a second order term, and that derivatives of $\alpha$ are tantamount to derivatives of $\omega$. Then,

$$
\begin{gathered}
-\frac{1}{2} \operatorname{Tr}\left[\frac{M^{2}}{-\partial^{2}+M^{2} \tilde{\omega}} \alpha \frac{M^{2}}{-\partial^{2}+M^{2} \tilde{\omega}} \alpha\right] \\
=-\frac{1}{48 \pi} \int d^{2} x \frac{1}{\omega^{2}} \partial_{\mu} \omega \partial^{\mu} \omega .
\end{gathered}
$$

This contribution has to be combined with the second order term coming from $J_{\text {reg }}^{(2)}$, which only differs in a $-\frac{1}{2}$ global factor. Then,

$$
\lim _{M \rightarrow \infty} J_{\text {reg }}[\eta, w, M]=-\frac{1}{96 \pi} \int d^{2} x \frac{1}{\omega^{2}} \partial_{\mu} \omega \partial^{\mu} \omega .
$$

which is the (Euclidean) Liouville action. Rotating back to Minkowski spacetime we obtain the result given in Eq. (9).

\section{The chiral anomaly}

This example shares many properties with the previous one of the conformal anomaly, and helps to understand the general nature of the procedure we have applied in Section 2.

We shall consider here $\mathscr{Z}[A]$, the vacuum functional for a massless fermion in $1+1$ dimensions,

$\mathscr{Z}[A]=\int[\mathscr{D} \bar{\psi} \mathscr{D} \psi]_{A} e^{i S_{F}[\bar{\psi}, \psi ; A]}$

with

$S_{F}[\bar{\psi}, \psi ; A]=\int d^{2} x \bar{\psi}(i \not-e A) \psi$.

Again, the integration measure depends on the background field configuration. The background gauge field $A$ may be decomposed as follows:

$e A_{\mu}=\partial_{\mu} \varphi+\epsilon_{\mu \nu} \partial_{\nu} \rho$

where $\varphi$ and $\rho$ are scalar and pseudoscalar fields, respectively. Then one easily sees that the fermionic action may be rewritten as

$S_{F}[\bar{\psi}, \psi ; A]=\int d^{2} x \bar{\psi}\left(i \not \partial-\not \partial\left(\varphi+\gamma_{5} \rho\right)\right) \psi$, 
what means that the gauge field may actually be erased by a gauge plus chiral transformation of the fermions. This, as for the previous example, implies that the classical dynamics of the system is trivial. However, the anomaly under chiral fermionic transformations introduces a non trivial quantum dynamics.

In the usual derivation, one performs chiral transformations of the fermions,

$\psi(x)=e^{i \alpha(x) \gamma_{5}} \psi(x)$,

$\bar{\psi}(x)=\bar{\psi}(x) e^{i \alpha(x) \gamma_{5}}$,

and the chiral anomaly appears from the non-invariance of the fermionic measure.

To consider an alternative derivation, we note that the Pauli-Villars regularized action in this case requires the addition of just one (massive) bosonic spinor field $\phi$, such that

$$
\begin{aligned}
S_{F}^{\mathrm{reg}}[\bar{\psi}, \psi, \bar{\phi}, \phi ; A]= & \int d^{2} x[\bar{\psi}(i \not \partial-e A) \psi \\
& +\bar{\phi}(i \not \partial-e A-M) \phi] .
\end{aligned}
$$

When $\alpha$ is a constant, the non-local infinitesimal chiral symmetry transformations of (35) are,

$\delta \psi=i \alpha \gamma_{5} \psi, \quad \delta \bar{\psi}=i \alpha \bar{\psi} \gamma_{5}$,

$\delta \phi=i \alpha \gamma_{5} \frac{\not D}{\not D-i M} \phi$,

$\delta \bar{\phi}=i \alpha \bar{\phi} \frac{\not D}{D-i M} \gamma_{5}$

where

$\not D=\partial+i e A$.

The action is invariant under these transformations, while the Jacobian becomes

$J=\operatorname{det}\left[1+i \alpha \gamma_{5}\left(1-\frac{D}{\not D-i M}\right)\right]^{-2}$

which may be rewritten as

$$
J=\exp \left[-2 i \alpha \operatorname{Tr} \gamma_{5}\left(\frac{1}{1+\frac{D^{2}}{M^{2}}}\right)\right] \text {. }
$$

The functional trace is finite, and reproduces the proper result when $M \rightarrow \infty$ :

$J=\exp \left[i \frac{e}{2 \pi} \alpha \int d^{2} x \epsilon^{\mu \nu} \partial_{\mu} A_{\nu}\right]$.

When $\alpha$ is spacetime dependent, the regularized action is no longer invariant. However, we may use that kind of transformation to get rid of the dependence in $A_{\mu}$. Those transformations are defined by

$\delta \psi=i \alpha(x) \gamma_{5} \psi, \quad \delta \bar{\psi}=i \bar{\psi} \gamma_{5} \alpha(x)$,

$\delta \phi=i \gamma_{5} \frac{\not D}{D-i M} \alpha(x) \phi$,

$\delta \bar{\phi}=i \bar{\phi} \alpha(x) \frac{D}{\not D-i M} \gamma_{5}$,

and the corresponding variation of the action is

$$
\begin{aligned}
\delta S_{F}^{\mathrm{reg}}= & -\int d^{2} x\left[\bar{\psi} \partial\left(\gamma_{5} \alpha\right) \psi\right. \\
& \left.+\bar{\phi} \partial\left(\gamma_{5} \alpha\right) \phi\right] .
\end{aligned}
$$

The Jacobian is easily shown to be

$J=\exp \left[i \frac{e}{2 \pi} \int d^{2} x \alpha(x) \epsilon^{\mu \nu} \partial_{\mu} A_{\nu}\right]$.

\section{Conclusions}

We have presented two concrete examples of systems where the regularized action has a non local symmetry which is the natural extension of the standard symmetry of the unregularized action. Moreover, the application of those transformations in the functional integral framework yields regularized Jacobians which properly reproduce the anomalies when the cutoff tends to infinity. This makes the connection between the regularization of the diagrams of a model, and the regularization of its Jacobian more transparent than in the usual setting.

We remark that our method differs also from performing the usual (local) transformations to the regularized action. This procedure would give no Jacobian, due to the cancellation between bare fields and regulators, while the regularized action would be non-invariant. The anomaly would appear in this case from the non-invariance of the regularized action. 
Finally, we want to remark that the phenomenon we have described, namely, the existence of a remnant of the classical symmetry for the regularized action is not new. It has been recently emphasized [7] that massless fermions on the lattice, even thought the regularization breaks the naive chiral symmetry, may have a lattice equivalence of that symmetry, if the lattice Dirac operator satisfies the Ginsparg-Wilson relation [8]. Indeed, this relation can be used to derive the chiral anomaly and the related index theorems on the lattice [9].

\section{Acknowledgements}

This work was supported by Universidad de Buenos Aires, CONICET (Argentina), Fundacion Antorchas, ANPCyT, CNEA and the Abdus Salam International Centre for Theoretical Physics. The authors would like to thank the Abdus Salam Inter- national Centre for Theoretical Physics for hospitality during completion of this work.

\section{References}

[1] K. Fujikawa, Phys. Rev. Lett. 42 (1979) 1195; Phys. Rev. D 21 (1980) 2848; for a more recent review see R.D. Ball, Phys. Rep. 182 (1989) 1.

[2] A.M. Polyakov, Phys. Lett. B 103 (1981) 207; see also K. Fujikawa, Phys. Rev. Lett. 44 (1980) 1733.

[3] A.M. Polyakov, Gauge fields and strings, Harwood Academic Publishers, 1987.

[4] S. Deser, M.J. Duff, C.J. Isham, Nucl. Phys. B 111 (1976) 45; P.C.W. Davies, S.A. Fulling, W.G. Unruh, Phys. Rev. D 13 (1976) 2720.

[5] C. Bernard, A. Duncan, Ann. Phys. 107 (1977) 201.

[6] I.J.R. Aitchison, C. Fraser, Phys. Lett. B 146 (1984) 63; Phys. Rev. D 31 (1985) 2605; B.L. Hu, D.J. O’ Connor, Phys. Rev. D 30 (1984) 743.

[7] M. Luscher, Phys. Lett. B 428 (1998) 342.

[8] P. Ginsparg, K. Wilson, Phys. Rev. D 25 (1982) 2649.

[9] K. Fujikawa, Nucl. Phys. B 546 (1999) 480; Phys. Rev. D 60 (1999) 074505. 